\title{
Alignment of the ATLAS Inner Detector tracking system
}

\section{Oleg BRANDT*}

University of Oxford and University of Göttingen

E-mail: oleg.brandtecern.ch

\begin{abstract}
The Large Hadron Collider (LHC) at CERN is the world largest particle accelerator. It will collide two proton beams at an unprecedented center of mass energy of $14 \mathrm{TeV}$. ATLAS is equipped with a charge particle tracking system built on two technologies: silicon and drift tube based detectors, composing the ATLAS Inner Detector (ID). The alignment of the tracking system poses a challenge as one should solve a linear equation with almost 36000 degrees of freedom. The required precision for the alignment of the most sensitive coordinates of the silicon sensors is just a few microns. This limit comes from the requirement that the misalignment should not worsen the resolution of the track parameter measurements by more than $20 \%$. So far the proposed alignment algorithms are exercised on several applications. We will present the outline of the alignment approach and results using real data from cosmic rays and large scale computing simulation of physics samples mimicking the ATLAS operation during real data taking. The full alignment chain is tested using that stream and alignment constants are produced and validated within 24 hours. Cosmic ray data serves to produce an early alignment of the real ATLAS Inner Detector even before the LHC start up. The impact of the alignment on physics measurements will be discussed.
\end{abstract}

European Physical Society Europhysics Conference on High Energy Physics

July 16-22, 2009

Krakow, Poland

\footnotetext{
* Speaker.
} 


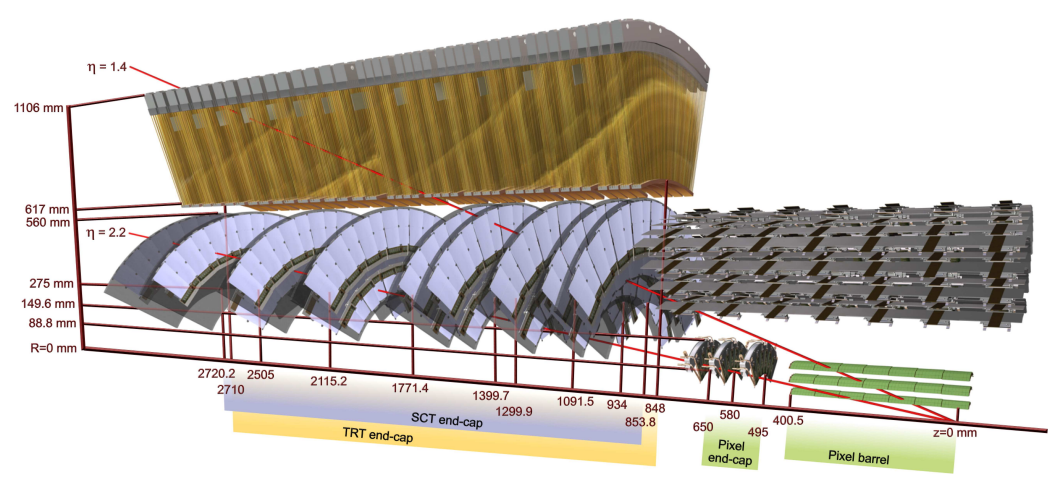

Figure 1: View of one segment of the Inner Detector of ATLAS with two tracks.

\section{The ATLAS Inner Detector}

The ATLAS Inner Detector (ID), depicted in Figure 1, is designed to measure the momentum of charged particles from their bending radius in the $2 \mathrm{~T}$ solenoidal magnetic field, and to provide a good pattern recognition for efficient vertexing. It comprises the following subdetectors [1, 2, 3]:

- The Pixel detector is a silicon tracking device with a nominal pixel size of $50 \mu \mathrm{m} \times 400 \mu \mathrm{m}$. It provides a 2-dimensional measurement with a nominal intrinsic resolution of $10 \mu \mathrm{m} \times$ $115 \mu \mathrm{m}$ in $R-\phi \times z^{1}$ for single-pixel hits [3]. The barrel part of the Pixel detector is comprised of three layers, whereas the endcaps consist of 3 disks each.

- The Semi-Conductor Tracker (SCT) is a silicon strip detector with 4 barrel layers and 9 endcap disks. Each barrel module features two single-sided back-to-back strip wafers with $80 \mu \mathrm{m}$ pitch, affixed at $40 \mathrm{mrad}$ stereo angle. At normal track incidence angle, this setup provides for a space point resolution of approximately $17 \mu \mathrm{m} \times 580 \mu \mathrm{m}$ in $R-\phi \times z[1,3]$.

- The Transition Radiation Tracker is a gaseous straw tube detector. It consists of approx. 300,000 individual straw tubes with a $4 \mathrm{~mm}$ diameter, providing a per-tube resolution of about $130 \mu \mathrm{m}$ in $R-\phi$ [3]. The straws of the TRT are arranged into 96 barrel modules and 2 $\times 20$ endcap wheels.

\section{The Alignment of the ATLAS Inner Detector}

The ID is an essential ingredient to achieve the ambitious physics goals of the ATLAS collaboration. However, its full potential can only be exploited with the exact knowledge of the positions of tracker modules and their orientation in space, i.e. $3+3$ Degrees of Freedom (DoF) per module. We aim to achieve an alignment quality sufficient as not to let of the track parameter resolution be degraded by more than $20 \%$ due to misalignments [1]. Studies with simulated Monte Carlo (MC) events show that this requirement translates into a precision about $7 \mu \mathrm{m}(12 \mu \mathrm{m})[30 \mu \mathrm{m}]$ in the critical $R-\phi$ direction for the pixel (SCT) [TRT] detector, which are about $\mathscr{O}(10)$ below the assembly tolerance. To achieve this level of precision, track-based alignment techniques will be employed: tracks fitted to particle trajectories, as measured by the ID in situ, are used to determine the position and orientation of its modules by optimising track-hit residual distributions.

Several track-based alignment algorithms were developed on ATLAS: three for the alignment of the silicon detectors and one for the TRT, see [4] and references therein. We have used them

\footnotetext{
${ }^{1}$ See [1] for a definition of the ATLAS coordinate frame.
} 


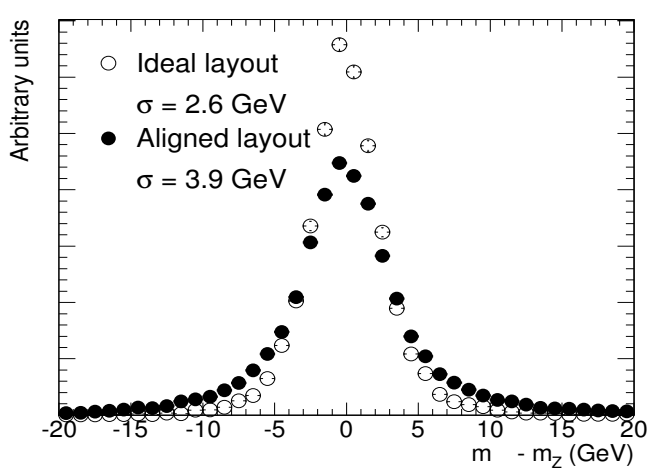

Figure 2: The difference $m_{\mu \mu}^{\text {reconstructed }}-m_{Z}$ for simulated $Z \rightarrow \mu \mu$ events where the muons have reconstructed in the ID only.

to align parts of the ID during commissioning in the Combined Test Beam setup [4] as well as on-surface with cosmic rays [5]. Moreover, these alignment algorithms were thoroughly tested in full scale with simulated MC collision events on several occasions. An impressive level of alignment precisison is achieved, as can be seen from e.g. the Figure 2 [3]: the contribution to the width of the $m_{\mu \mu}^{\text {reconstructed }}-m_{Z}$ peak from remaining misalignments is only about as large as from Multiple Coulomb Scattering (MCS), over the entire $|\eta|<2.5$ range of the ID.

\section{Alignment of the ATLAS Inner Detector with 2008 Cosmic Ray Data}

All 3+1 alignment algorithms at ATLAS were employed to align the entire ID in situ in the ATLAS cavern using the cosmic ray data collected in autumn 2008, the so-called M8+ run. During this period, about $880 \mathrm{k}(2 \mathrm{M})$ tracks with SCT hits and about $190 \mathrm{k}(230 \mathrm{k})$ tracks with pixel hits were recorded with solenoid magnetic field on (off). Figure 3 demonstrates the track-hit residual distributions reconstructed using the pixel and SCT detectors in five representative runs of the ID with solenoid on (about $25 \%$ of M8+ data). Only residuals in the barrel region from tracks with $p_{\mathrm{T}}>2 \mathrm{GeV},\left|d_{0}\right|<50 \mathrm{~mm}$, and $\left|z_{0}\right|<400 \mathrm{~mm}$ were used. Track-hit residual distributions before (after) alignment with the GLOBAL $\chi^{2}$ algorithm are shown in black (blue). The distribution for simulated MC events, reconstructed with full ID geometry knowledge, is shown in red. The degradation of the residual resolution due to misalignments is comparable in magnitude to the degradation due to MCS. The track-hit residual distribution for the TRT detector before (black) and after (blue) alignment is presented in the same Figure. Here, tracks with $p_{\mathrm{T}}>2 \mathrm{GeV}, N_{\text {pixel }}^{\text {hit }}>2$, $N_{\mathrm{SCT}}^{\text {hit }}>9, N_{\text {TRTbarrel }}^{\text {hit }}>45$ were used, i.e. their residuals are sensitive both to the internal TRT misalignments, as well as to the relative misalignment of the TRT with respect to the silicon tracker.
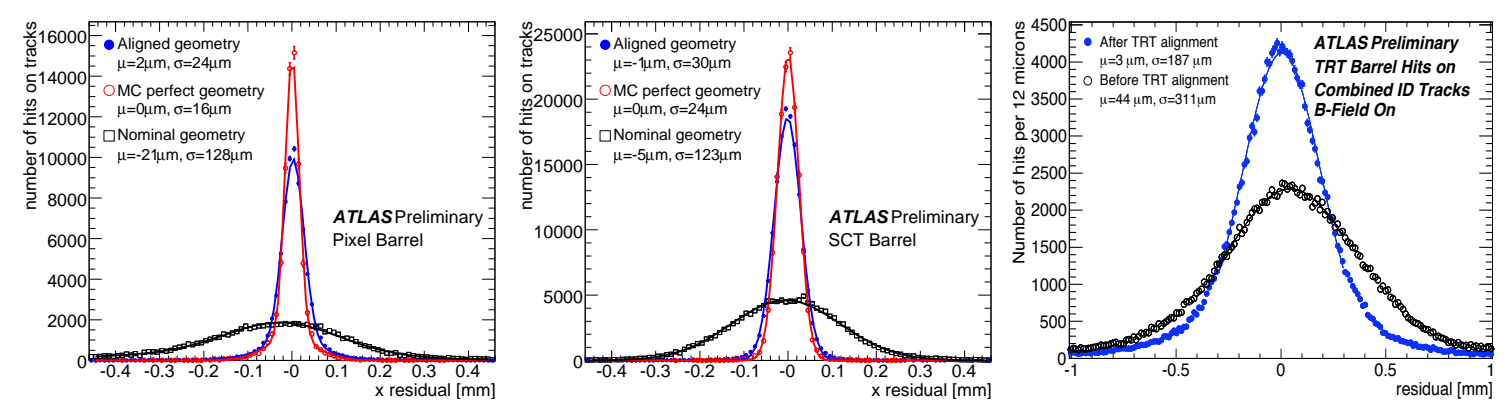

Figure 3: Track-hit residuals in $R-\phi$ for the pixel (left), SCT (middle), and TRT (right) detectors as found in M8+. See text for details. 

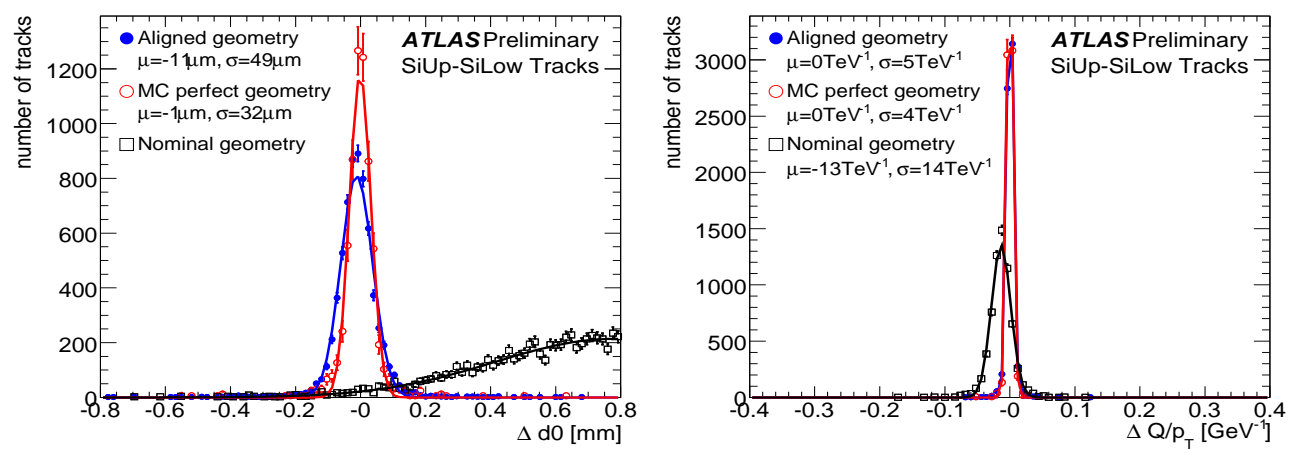

Figure 4: $\Delta d_{0} \equiv d_{0}^{\text {upper }}-d_{0}^{\text {lower }}\left(\right.$ left) and $\Delta Q / p_{\mathrm{T}} \equiv Q / p_{\mathrm{T}}^{\text {upper }}-Q / p_{\mathrm{T}}^{\text {lower }}$ (right) distributions for cosmic ray tracks split into an upper and lower segment as found in M8+. See text for details.

We evaluated the track parameter reconstruction using the following procedure: a cosmic ray track is split near the interaction point, and its upper and lower segments are refitted independently. This allows for a direct comparison of their track parameters. The resulting $\Delta d_{0} \equiv d_{0}^{\text {upper }}-d_{0}^{\text {lower }}$ and $\Delta Q / p_{\mathrm{T}} \equiv Q / p_{\mathrm{T}}^{\text {upper }}-Q / p_{\mathrm{T}}^{\text {lower }}$ distributions for the silicon tracker are shown in Figure 4 . In addition to the cuts above, $N_{\text {pixel }}^{\text {hit }} \geq 3, N_{b \text {-layer }}^{\text {hit }} \geq 1$, and $N_{\text {silicon }}^{\text {hit }} \geq 7$ are required for each segment. The $\Delta d_{0}$ distribution, crucial for vertexing, improves dramatically: being centred at about $0.8 \mathrm{~mm}$ before alignment, the $\mu(\sigma)$ parameters of a Gaussian fit are $-11 \mu \mathrm{m}(49 \mu \mathrm{m})$ for aligned geometry. A similarly dramatic improvement is observed in the $\Delta Q / p_{\mathrm{T}}$ distribution: it indicates an almost bias-free reconstruction of the signed track curvature $Q / p_{\mathrm{T}}$ after alignment.

Figures 3 and 4 demonstrate a high quality of alignment of the ATLAS ID achieved using cosmic ray data collected in the M8+ run. It is notably better for the upper and lower barrel quadrants of the respective detectors. This is because of their better illumination and thus smaller statistical uncertainties on the alignment constants. The distrubutions shown are not directly transferrable to collision data: well-aligned parts of the detector find an overproportional contribution, and other parameters, e.g. the incidence angle of tracks, their momentum spectrum, etc. are different.

\section{Alignment Prospects of the ATLAS Inner Detector with Collision Data}

The impact of misalignments on early ATLAS physics was investigated using MC simulations. For this, two alignment scenarios - "Day-1" and "Day-100" - were defined. As the naming suggests, they refer to the alignment quality expected on the $1^{\text {st }}$ and $100^{\text {th }}$ day of LHC collisions. Logically, Day-1 constants will be derived with cosmic ray data only and thus should be compatible with the M8+ alignment results. In particular, this implies a better alignment quality in the barrel compared to the end-caps due to illumination. On the contrary, Day-100 constants will be determined using a combination of cosmic ray and collision data, and are likely to result in a more uniform alignment quality across the individual subdetectors of the ATLAS ID. To define the Day1 and Day-100 misaligned geometries, the module positions were smeared with a Gaussian. The $\sigma$-parameters chosen are summarised in Table 1.

We evaluated the impact of Day-1 and Day-100 misalignments on ATLAS physics performance using MC simulations [6]. Among other properties, their effect on tracking and vertexing efficiency, $b$-tagging, and the momentum reconstruction has been studied. Figure 5 shows the dimuon mass distribution for simulated $Z \rightarrow \mu \mu$ and $J / \Psi \rightarrow \mu \mu$ events. The muons were recon- 


\begin{tabular}{l|cc|cc}
\hline Detector & Day-1: Barrel & Day-1: End-Cap & Day-100: Barrel & Day-100: End-Cap \\
\hline \hline Pixel & 20 & 50 & 10 & 10 \\
SCT & 20 & 50 & 10 & 10 \\
TRT & 100 & 100 & 50 & 50 \\
\hline
\end{tabular}

Table 1: The $\sigma$-parameters for Gaussian smearing of ID module positions used for generating Day-1 and Day-100 misaligned geometries. All values are in $\mu \mathrm{m}$.
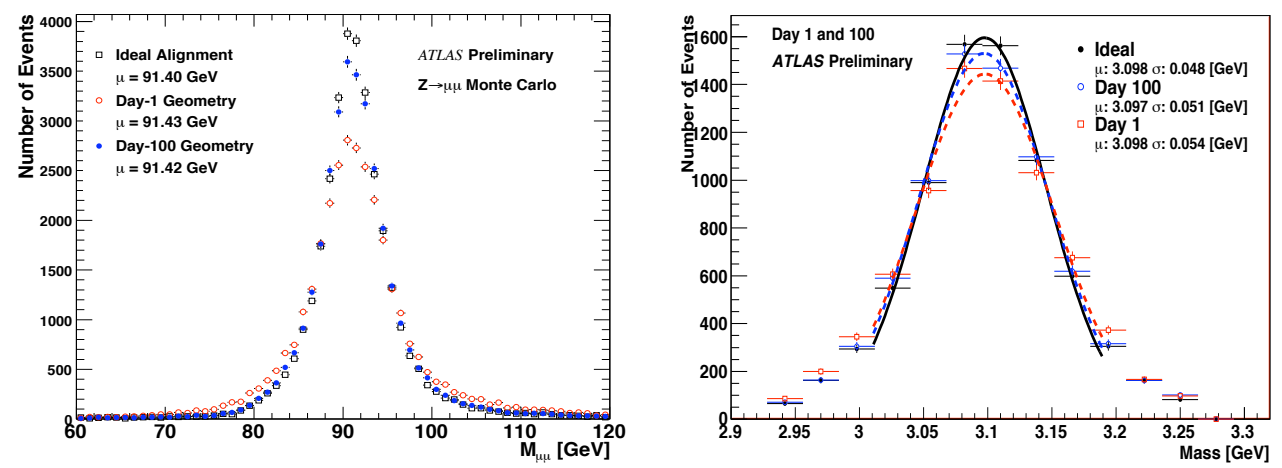

Figure 5: The reconstructed dimuon mass $m_{\mu \mu}$ for simulated $Z \rightarrow \mu \mu$ (right) and $J / \Psi \rightarrow \mu \mu$ (left) events. The muons have reconstructed using the ID only. See text for details.

structed in the ID only using the perfectly known (black), the Day-1 (red), and the Day-100 (blue) geometries. For $Z \rightarrow \mu \mu$ events, the width of the $m_{\mu \mu}$ peak deteriorates by about $50 \%$ (13\%) for Day-1 (Day-100) geometry. Analogously, about 13\% (6\%) is found for $J / \Psi \rightarrow \mu \mu$. The different impact of misalignments on the two physics processes is expected: it is the uncertainty on the curvature and not the momentum measurement that is relevant here.

\section{Conclusion}

The infrastructure for track-based alignment of the ATLAS Inner Detector is in a good shape for first LHC collisions. 3+1 alignment algorithms are in place, and were validated using simulated MC events. We derived a preliminary set of alignment constants using the cosmic ray data collected during the M8+ run in autumn 2008. Its performance is very promising and will be further improved with cosmic ray data collected in 2009. Further, we evaluated the impact of misalignments on early ATLAS physics using MC simulations, and found that they do not significantly compromise the physics goals of our collaboration for 2009-2010.

\section{References}

[1] The ATLAS collaboration, CERN/LHCC 97-16/17, 1997.

[2] The ATLAS collaboration, CERN/LHCC 99-14, 1999.

[3] ATLAS Collaboration, J. of Inst. 3 (2008) S08003.

[4] A. Ahmad et al., J. of Inst. 3 (2008), no. 09, P09004.

[5] E. Abat et al., J. of Inst. 3 (2008), no. 08, P08003.

[6] The ATLAS collaboration, ATL-PHYS-PUB-2009-080, 2009. 